\title{
IDEAS SOBRE LA SALUD Y EL AIRE AMBIENTAL. UN ESTUDIO COMPARATIVO ENTRE TEXTOS MÉDICOS ANTIGUOS Y MEDIEVALES
}

\author{
Jaime E. Bortz \\ Claudia E. Sedlinsky
}

\section{Introducción}

La personalidad de Maimónides (Córdoba, España, 1138; Fustat, Egipto, 1204) es considerada un paradigma de las ciencias y humanidades del mundo árabe medieval pues en su figura se conjugaron la filosofía, la ciencia y la teología de su época.

Sus escritos médicos reconocen diversas influencias: la herencia de la Antigüedad clásica grecorromana, la medicina árabe medieval y las fuentes hebreas bíblicas y postbíblicas.

El interés en el estudio de los escritos médicos de Maimónides (en adelante: MM) es creciente pues, a través de ellos, es posible trazar un panorama de la formación científica en el mundo de lengua árabe de la segunda mitad del siglo XII, período en el cual la importancia de la medicina islámica comienza a declinar.

En el curso de los últimos cuarenta años se ha incrementado la cantidad de estudios sobre MM y su abundante producción escrita, revelando un interés creciente en sus textos y en los textos de su época. Durante este 
período se han publicado ediciones críticas de ocho de las diez obras médicas del cordobés, de lo cual ha resultado que gran parte de esta nueva bibliografía se refiere a los aspectos médicos de su obra. Sin embargo, restan aspectos de su producción escrita que no han sido suficientemente estudiados aún. Uno de ellos consiste en la relación entre la adhesión de MM a las tradiciones médicas que le precedieron y la cuota de originalidad científica que le tocó a él aportar. Esta relación puede ser estudiada sólo si se compara la posición de MM sobre un aspecto científico puntual con las posiciones de tradiciones científicas anteriores sobre el mismo aspecto. De esta forma es posible estudiar los fenómenos de tradición y cambio en el desarrollo de la historia de la ciencia desde la Antigüedad hasta la Edad Media.

En el presente trabajo se estudiarán los fenómenos de tradición y cambio relativos a la influencia del aire ambiental y del viento en los procesos de salud y enfermedad de los seres humanos, realizando un análisis comparativo de textos maimonitas, grecorromanos, bíblicos y talmúdicos.

\section{Los textos de Maimónides}

En el cuarto capítulo del Régimen de Salud aparece el siguiente párrafo:

"Primero es necesario cuidarse de mejorar el aire, luego de mejorar el agua y luego los alimentos. Esto es así porque lo que los médicos llaman pneumas son vapores ligeros que se encuentran en el cuerpo de los seres vivientes... Su origen y sustancia fundamental deriva del aire inhalado del exterior... El vapor que se encuentra en la sangre del corazón y los vasos pulsátiles se llama espíritu vital; y el vapor que se encuentra en las cámaras del cerebro y que desde allí se disemina, a través de las oquedades de los nervios, se llama espíritu psíquico. La fuente de todos ellos y el grueso de su substancia deriva del aire que es inhalado desde el exterior. Y si este aire es rancio, pútrido o turbio, todos los espíritus se alteran y sus características se transforman en lo opuesto de lo que deberían ser.

Galeno dijo: se debe prestar atención a la calidad del aire que se inhala al interior del cuerpo para que el cuerpo esté perfectamente balanceado y libre de todo lo que pueda contaminarlo.

El autor dice: cuanto más delicado es el pneuma, más susceptible es a los cambios del aire. El espíritu natural es más espeso que el vital, y el vi- 
tal es más espeso que el espíritu psíquico. Con un pequeño cambio en el aire aparece un cambio reconocible en la condición psíquica. Por ello existen muchas personas cuyas actividades psíquicas declinan por la contaminación del aire; desarrollan confusión del pensamiento, debilidad de comprensión y pérdida de memoria, aún cuando sus actividades vitales y naturales no experimentan ningún cambio'notorio.

Comparar el aire de las ciudades con el aire del desierto y los bosques es como comparar las aguas espesas y turbias con las puras y claras. Esto es porque las ciudades, por la altura de sus edificios, la estrechez de sus calles, la basura proveniente de sus habitantes, sus residuos, sus muertos, las deyecciones de sus animales y la putrefacción de sus alimentos todo ello hace que el aire sea estancado, turbio, vaporoso y espeso. El pneuma también se transforma en forma acorde pero la persona no percibe lo que ha ocurrido con él. Si no se tiene posibilidades de elegir en este aspecto - porque se ha crecido en áreas urbanas y uno se ha acostumbrado a ellas- se debe elegir, de entre las ciudades, aquella que tenga amplios horizontes, preferentemente orientados hacia el norte y el este, y que esté alta en las montañas, donde los bosques son ralos, con pocos árboles y poca agua. Y si aún así uno no puede elegir, o sea, no puede mudarse de la ciudad, por lo menos trate de morar en los suburbios orientados hacia el norte y el este. Los cuartos deben estar localizados en un edificio alto con amplio patio para que el viento del norte y el sol puedan atravesarlos; porque el sol disuelve la putrefacción del aire, lo fluidifica y lo purifica. Debe ponerse atención en ubicar los baños tan lejos de los cuartos como sea posible; y también se debe tratar de mejorar el aire y secarlo con perfumes y fumigantes, en forma apropiada y de acuerdo con los cambios en el aire. Esto es fundamental para comenzar un régimen de cuidados de la salud del cuerpo y del alma.» (1).

Un pasaje que trata del mismo tema aparece también en el capítulo decimotercero del Tratado del Asma:

«Dice el autor: cuanto más delicado es el pneuma, más afectable es por el aire circundante. Así el espíritu natural es más denso que el vital, y éste es, a su vez, más denso que el animal. Cada cambio en el aire circundante produce una alteración en el espíritu animal. Por ello, algunas personas, enfrentadas a un cambio climático repentino, experimentan una disminución en sus funciones animales, una forma de estupor, la pérdida de la capacidad de razonar o de la memoria, mientras que los espíritus natural y vital no se alteran. La relación entre el aire de la ciudad y sus calles (por un lado) y el (aire) que se puede hallar en un sitio abierto 


\begin{abstract}
puede ser comparada con la relación entre el agua sucia y groseramente contaminada y su contraparte clara y límpida. El aire de la ciudad es estancado, turbio y denso, resultado natural de sus edificios altos, calles estrechas, los desechos de sus habitantes, los cadáveres y esqueletos de sus animales, alimentos en mal estado y cosas similares. Los vientos llevan sigilosamente este aire hacia adentro de las casas y muchos hombres se enferman sin darse cuenta de ello. Sin embargo, si parece imposible escaparse (de la ciudad) por haber crecido en una urbe y estar acostumbrado a sus caminos, uno debe, al menos, elegir residencia ubicada en un sitio abierto, que mire hacia el nordeste, preferentemente sobre la ladera de una montaña escarpada y boscosa, y lejos de charcas y pantanos. Si fuera imposible mudarse a otra ciudad, lo que seguiría (en orden decreciente de importancia) es residir en un suburbio, al sur o al norte. Las salas de estar están mejor localizadas en el cuarto superior, mirando hacia una calle ancha expuesta al viento del norte y al brillo del sol , ya que la luz solar barre el aire malo y lo transforma en aire claro y límpido. Los baños deben situarse tan lejos de las salas de estar como sea posible. El aire debe ser mantenido siempre seco con esencias dulces, fumigación y agentes desecantes. El mantenimiento del aire fresco es la regla más importante para la preservación de la salud del cuerpo» (2).
\end{abstract}

Ambos textos son casi idénticos en sus ideas, por lo que se puede presumir que uno fue copiado del otro (3). La única diferencia es que el pasaje que figura en el Tratado finaliza con una sentencia que no se registra en el Régimen:

«El mantenimiento del aire fresco es la regla más importante para la preservación de la salud del cuerpo.»

En otro pasaje del mismo Tratado se apunta que:

«Es bien sabido que el aire limpio y puro, libre de de toda contaminación, es aconsejable para todas las personas, ya sea sanas o enfermas» (4).

Las alteraciones en la composición del aire pueden ser causa de graves trastornos de la salud, sostiene MM. Esto sucede por dos mecanismos diferentes. El primero de ellos es la alteración de los pneumata. De acuer- 
do con la fisiología de la época, en el cuerpo de los seres vivos existirían vapores denominados pneumata o spiritus (5). Existirían tres clases de pneumata, a saber:

* El pneuma natural.

* El pneuma vital.

* El pneuma psíquico.

Como todos ellos se crearían a partir del aire exterior, las modificaciones en la calidad del aire alterarían la calidad de los pneumata. Cuando más delicado es el pneuma, más sensible es a los cambios del aire; si el aire exterior es rancio, pútrido o turbio, los pneumata se alteran y el individuo puede enfermar.

Maimónides recoge en sus Aforismos Médicos de Moisés la siguiente sentencia que hace suya:

"Se debe tratar de normalizar en forma temprana el espíritu de estos tres órganos. Para (mejorar) la calidad del espíritu físico, se debe mejorar el aire externo, o abrir los poros de la piel, o purificar las vías respiratorias de secreciones espesas y viscosas... (Para mejorar) el espíritu natural, se debe consumir alimentos y bebidas que sean nutritivos, beneficiosos y poco perjudiciales» (6)

El segundo mecanismo por el cual las modificaciones en el aire inhalado producirían trastornos de la salud consistiría en las alteraciones del calor corporal, innato o natural. Este concepto fue uno de los cuatro pilares de la fisiología de Galeno y de los galenistas medievales, tal como lo apuntara Laín Entralgo (7). Véase el siguiente párrafo:

"Si el cuerpo se torna anormal porque se llena de secreciones, alimentos o bebidas, y no existe un cuarto para que el aire inhalado encuentre su camino, se produce estrangulamiento del calor innato... El calor innato fluye muy lentamente y se extingue $\mathrm{y}$, como resultado, aparece rápidamente la muerte... Las causas que afectan al calor innato desde el exterior son seis... la quinta es resultado del deterioro de su sustancia... Esto sucede de dos formas. Primero, por la inspiración de aire frío mezclado con gases perjudiciales y con mal olor, como en el caso de los gases que emanan de cadáveres y los gases emitidos por cloacas y pozos ciegos, en 
los cuales se encuentra fango negro y fétido. Así, la sustancia del calor natural se deteriora. Muchas personas han muerto repentinamente por los gases desprendidos de cloacas y muros en los cuales el polvo sucio se ha descompuesto" (8).

MM sostenía también que el viento influye sobre los procesos de salud y enfermedad. En sus aforismos recoge y hace suyas las siguientes reflexiones:

«El peor (clima) de cada país es el cercano a los vientos del este, y donde prevalecen los vientos desapacibles y fríos» (9).

«El mejor viento es el que sopla desde el litoral. El siguiente es el que sopla desde las montañas. El peor de estos (vientos) es el que viene de los ríos, de lagos o de campos. El intermedio entre éstos es el que viene de otras (direcciones)» (10).

A continuación se examinarán las opiniones de los médicos grecorromanos sobre el mismo tema.

\section{Los textos grecorromanos}

El tratado Aires, Aguas, Lugares es, de los textos del Corpus Hippocraticum, el que mayor cantidad de material aporta para el presente estudio. Este texto es, en realidad, un manual práctico destinado a dar consejos útiles a los griegos que se asentaban en nuevas colonias en sitios desconocidos de la cuenca del Mediterráneo (11).

Como se sabe, la ciencia hipocrática aceptaba la existencia de cuatro humores corporales (chymoi), llamados bilis blanca o flema, bilis negra, bilis amarilla y bilis roja o sangre. Estos humores no eran concebidos como intrínsecamente patógenos en tanto se mantuvieran en las proporciones adecuadas (eukrasia); sin embargo, el desequilibrio en la concentración de uno de ellos (diskrasia) sería causante de enfermedad. Estas ideas se aplicaban a todas las áreas de la medicina y también a la climatología médica. Se pensaba que cada humor era afectado por una condición climática diferente en forma casi exclusiva: 
«La ciudad que están expuesta a vientos cálidos... (en ella) sus habitantes tienen cabezas húmedas y llenas de flema... » (12).

"Y ésta es la condición de las ciudades en situación opuesta, que reciben vientos fríos... ellos (los habitantes) deben ser más biliosos que flemáticos... (13)

Estos dos fragmentos ejemplifican una de las características de la medicina hipocrática: una visión bastante esquematizada del medio ambiente que refleja la predilección de los griegos clásicos por la categorización neta. En el mundo hipocrático, las enfermedades que se observan en un clima gobernado por vientos cálidos no aparecen en regiones de vientos fríos. Sería muy raro encontrar una enfermedad que se manifestara en regiones de climas opuestos. Las ciudades cuyos vientos fueran tibios —ni muy cálidos ni muy fríos- eran consideradas más saludables debido a la variedad climática experimentada.

«Las ciudades orientadas hacia el nacimiento del sol (14) son probablemente más saludables que las que miran hacia el norte y que las que están expuestas a vientos cálidos... » (15).

"Las ciudades orientadas hacia el ocaso del sol (16) y barridas por los vientos del este... estas ciudades son poco saludables» (17).

Se sabe también que la escuela médica de los neumáticos, fundada alrededor del año 50 d.C. puso especial énfasis en la acción del aire en el cuidado de la salud. Para ello, estos médicos estudiaron el aire de los valles, los pantanos y la orilla del mar, y su efecto sobre las enfermedades (18). Es Galeno, sin embargo, quien elevó a la categoría de dogma las creaciones de la medicina hipocrática. En el área de la climatología médica, Galeno se limitó a reforzar las opiniones que le precedieron sin producir innovaciones de valor, aunque desarrolló algunos corolarios a base de las ideas de Aguas, Aires, Lugares. Galeno es más específico cuando habla de la relación entre el aire y la construcción de ciudades:

«Es deseable respirar el mejor aire; esto vale para todas las edades de la vida. Considero que el aire de mejor calidad es el que es absolutamente puro. Y aire puro es el que no proviene de estanques, pantanos, o pozos, que desprenden un vapor perjudicial. También es tóxico el aire que pro- 
viene de ... grandes ciudades y de áreas densamente pobladas; y el que está contaminado por putrefacción de animales, vegetales, aceites o estiércol ... y el que, en un valle rodeado por montañas, no recibe brisa. Este aire es sofocante y viciado, como el de algunas casas cerradas, donde se conjugan la putrefacción y la falta de ventilación. Este aire es perjudicial para (personas de) toda edad; mientras que el aire absolutamente puro es beneficioso para todas las edades". (19).

\section{Los textos bíblicos y talmúdicos}

Una de las claves para entender los pronunciamientos de la antigüedad hebrea con respecto al mundo de la naturaleza radica en el precepto bíblico del bal tashjit, "no destruirás en vano". La fuente bíblica de este mandato es Deuteronomio 20:19, donde la divinidad prohibe la destrucción innecesaria de árboles en tiempos bélicos. Este principio fue invocado frecuentemente por los rabinos del Talmud para aplicarlo a la utilización instrumental del aire, del agua y de la tierra. La polución de estos tres elementos naturales por parte de los hombres es vista como la violación del mandato divino del bal tashjit y, por lo tanto, no es considerado sólo un acto de insensatez, sino también de sacrilegio.

Los rabinos emitieron ordenanzas bastante estrictas con respecto al aire de los países extranjeros. Antes de la destrucción del Segundo Templo de Jerusalén (70 d.C.) se emitió un decreto por el cual se consideraba ritualmente impuro el aire de los territorios ubicados fuera de la tierra de Israel. Se han sugerido varias razones para esta ordenanza. Es posible que los rabinos hayan pensado que la impureza se debería a los cuerpos insepultos de los ahogados en el Diluvio (20). Quizá los haya motivado el hecho que, en dicha época, la mayor parte de los judíos moría fuera de la tierra de Israel. También es posible que esta declaración de impureza ritual no se haya debido a motivos religiosos sino económicos y sociales, en un deseo de los líderes de la época en pro de desalentar la emigración masiva de judíos desde Israel en un período en el cual la situación general del país era muy difícil.

El aspecto más notable de las enseñanzas rabínicas en relación con los efectos del aire sobre la salud es la creación de la noción de mazikin (21). La Mishná (22) relata su creación: 
«Diez fueron las cosas creadas la víspera del Sábado, hacia el crepúsculo, a saber:

- El abismo (que englutió a Koraj y sus adherentes).

- El pozo (que proporcionaba agua a los judíos durante su travesía por el desierto).

- La boca de la burra del Bil'am (que habló a Bil'am el brujo).

- El arco iris.

- El maná.

- La vara de Moisés.

- El shamir (23).

- La escritura.

- El texto de las Tablas (de la ley).

- Las Tablas (de la ley).

Algunos solían agregar:

- Los mazikin.

- La tumba de Moisés.

- El cordero de Abraham nuestro patriarca (que sustituyó a Isaac en el sacrificio).

Otros agregan:

— Las primeras tenazas que se utilizaron para forjar otras.» (24)

Los mazikin eran una suerte de espíritus o demonios capaces de causar enfermedades y diseminarlas a través de sus desplazamientos por aire y agua. Se pensaba que a mediados del verano estos espíritus se volvían más activos por efecto del aire cálido; por lo tanto este período era visto como particularmente insalubre.

Los rabinos del Talmud destacaron los efectos saludables del aire de los sitios elevados. Se puede encontrar aquí nuevamente una motivación religiosa para una opinión médica. En el pensamiento judío clásico, los sitios elevados eran considerados como favorecidos por Dios porque sólo los lugares altos se salvaron de las aguas del Diluvio. Sin embargo, una fuente talmúdica relata que cuando un sabio se enfermó fue trasladado hasta la ciudad de Sephoris para recobrarse inhalando el aire puro del lugar (25) Por lo tanto, el aire de los lugares montañosos volvería a éstos 
saludables por sí mismos, independientemente de consideraciones teológicas.

La ciudad de Jerusalén ocupaba una posición destacada en los ojos de los judíos piadosos; por ello los rabinos se preocuparon por mantener y fomentar la pureza del aire de la ciudad. Se quemaban leños de árboles de canela para lograr un aroma placentero del aire:

«Rahabah dijo en nombre de Rabí Judá: los leños que se quemaban en Jerusalén eran del árbol de canela. Y cuando eran encendidos, su fragancia cubría toda la tierra de Israel» (26).

El aire de otras ciudades también fue protegido mediante ordenanzas rabínicas. Una ordenanza de la Mishná prescribe la obligación de mantener a una distancia de cincuenta cúbitos de cada ciudad las tumbas, los animales carroñeros y las curtiembres, en razón de los olores nauseabundos y potencialmente peligrosos que despiden (27). La Mishná (28) se ocupó también de las máquinas de trillar grano:

sis

«Las trilladoras deben ser colocadas a una distancia de cincuenta cúbitos de la ciudad. Ninguna persona puede colocar una trilladora en su domicilio a menos que haya alrededor un espacio libre de cincuenta cúbitos» (29).

Sobre esta fuenta la Guemará (30) se interroga y responde:

"¿Por qué debe mantenerse la trilladora a cincuenta cúbitos de la ciudad? Para impedir que ocasione daño. Podría esparcir la paja a través del aire» (31).

Análisis de los documentos

En el pensamiento de MM existen tres cosas que deben vigilarse para asegurar el correcto cuidado de la salud. En orden de importancia, de mayor a menor, ellas son: el aire, el agua y los alimentos. La vigilancia de 
las condiciones del aire es, en consecuencia, una de la principales tareas de promoción de la salud.

Las alteraciones en la composición del aire pueden ser causa de graves trastornos. Esto sucede por dos mecanismos diferentes: la alteración de los pneumata y la alteración del calor natural.

Los pneumata son, en la fisiología general galénica, uno de los cuatro conceptos fundamentales, junto con el alma ( $p s y k h e ́)$, la potencia o facultad (dynamis), y el calor natural (émphyton thermón) (32). Los pneumata son sustancias materiales sutiles y delicadas, por las cuales las potencias de las partes orgánicas pasan a ser la acción y el acto de ellas; es decir, son los instrumentos agentes del movimiento vital. La medicina galénica sostendrá la existencia de tres tipos de pneumata: el físico (o natural), el vital y el psíquico. Temkin ha demostrado que en los escritos de Galeno la división no es tan tajante y clara; esta división parece ser obra de los galenistas posteriores (33). El hígado es el sitio del pneuma natural, el órgano que crea la sangre, y el responsable de alimentar y nutrir a todo el cuerpo incluyendo al lado derecho del corazón. El corazón es el sitio del pneuma vital, creado a partir del pneuma traído traído desde los pulmones hasta el lado izquierdo del corazón; de allí se disemina por todo el cuerpo a través de los vasos pulsátiles (arterias). El pneuma psíquico se localiza en los ventrículos cerebrales, siendo el cerebro el asiento del alma y el órgano que controla las funciones vitales corporales. MM sostuvo que, de los tres pneumata, el psíquico sería el más delicado y el más susceptible de modificaciones; en consecuencia, el más influenciable por cambios en el aire inspirado. Alteraciones imperceptibles del aire exterior serían capaces de crear trastornos en el pneuma psíquico, incluso sin afectar las funciones naturales y vitales. Es aquí donde deben insertarse las prevenciones sobre la impureza, estancamiento y turbidez del aire que MM formuló en el Régimen de Salud y en el Tratado del Asma.

Existe otro mecanismo por el cual una persona puede ser dañada como consecuencia de la alteración del aire ambiental. Se trata de un cambio en las condiciones del calor natural, innato o corporal. Como fue dicho más arriba, fue uno de los cuatro conceptos fundamentales de la fisiología galénica. No se conoce aún el origen primigenio de este concepto. Laín Entralgo ha sostenido que se trata de una vieja doctrina perviviente en Galeno (34); de lo cual se desprendería que es muy anterior al médico de Pérgamo. García Ballester ha sostenido que el calor innato fue un concepto diferente de los pneumata (35); Müntner, en cambio, 
sostenía que el calor natural era concebido como parte del pneuma vital (36).

MM sostiene -basándose en Galeno- que el aire ambiental corrupto deterioraría el sustrato de la respiración y podría causar el síncope o colapso. Adjudica este fenómeno al deterioro en el calor innato o natural. También sostiene que el inspirar aire frío mezclado con gases y malos olores - como los que emanan de residuos biológicos- deteriora la sustancia del calor natural y la persona, en consecuencia, se enferma.

MM aportó una vivida descripción de las ciudades egipcias de su tiempo, y estableció relaciones entre la situación urbanistica y el cuidado de la salud. Los poblados se caracterizaban por tener un aire espeso y turbio debido a la altura de los edificios, la estrechez de sus calles y los residuos provenientes de los pobladores: basura de diversa especie, cadáveres de animales, deyecciones y alimentos putrefactos. Por este motivo el autor sugirió morar en los suburbios de la ciudad orientados hacia el norte y el este; ubicar los cuartos para dormir en un edificio alto, con amplio patio, para que el viento del norte y el sol puedan atravesarlos; ubicar las letrinas lejos de los cuartos, y mejorar el aire con esencias y fragancias. En la opinión de MM, el lugar ideal para vivir sería una ciudad con amplios horizontes hacia el norte y el este, localizada en lo alto de una montaña, pues el aire puro y límpido de ese sitio sería el más aconsejable para las personas.

Las ideas maimonitas sobre la relación del aire ambiental con la salud se originan en la medicina grecorromana y en las fuentes rabínicas. El escrito hipocrático Aires, aguas, lugares estaba dedicado al estudio de la influencia del clima sobre la salud, en su primera parte, y del clima sobre la inteligencia, en la segunda. Las ideas hipocráticas sobre climatología médica influyeron notablemente en el mundo antiguo. Aires, aguas, lugares es, en realidad, un manual de medicina que trata sobre el afrontar situaciones médicas desconocidas, y está dirigido a los médicos de las colonias griegas de la Hélade. En los textos hipocráticos los humores son afectados por el ambiente en general, y cada uno de ellos por otra condición particular del tiempo. Las sectas médicas que surgieron a partir de la escuela hipocrática recogieron esta doctrina de climatología médica junto con la doctrina de los humores. Una de las sectas más tempranas, los empíricos, estaba muy interesada en la conservación del ambiente y sostenía que el tratamiento médico debe ser hábitat-específico, es decir, con particularidades para cada ambiente; y que por lo tanto el 
médico debía tener un acabado conocimiento de las condiciones locales al prescribir un régimen al paciente. La escuela neumática, con Ateneo de Atalia (c. 50 d.C.) y Areteo de Capadocia (S. I y II d.C.) enfatizó la acción del aire sobre el pneuma, y estudió el efecto de diversos tipos de aire sobre la salud. Galeno (s. II d.C.), por su parte, elevó la teoría ambiental hipocrática y la doctrina de los humores a la categoría de verdad absoluta.

La medicina hipocrática mostró dos características relevantes en lo que a su visión del ambiente se refiere. En primer lugar, tuvo una visión holística en la que el agua, el aire y el lugar estaban íntimamente conectados con la salud. Segundo, tenía una visión esquematizadora del ambiente que reflejaba la predilección griega por la categorización neta y rotunda: las enfermedades que se observan en los lugares con vientos calientes no se observan en lugares donde soplan vientos fríos y viceversa; las ciudades con vientos templados son más sanas por la variedad del clima.

El cuidado del aire ambiental también está desarrollado extensamente en las fuentes talmúdicas, aunque no tan bien ordenado y sistematizado como en las grecorromanas. El Talmud se apoya en una cita bíblica (37) para elaborar la doctrina del bal tashjit, por lá cual se prohibe la destrucción innecesaria de recursos naturales en tiempos de guerra. Elevado a la categoría de una prescripción religiosa, el poluir el aire, el agua o la tierra es visto como una violación del precepto bal tashjit y, por lo tanto, como un acto sacrílego además de necio. Los rabinos del Talmud invocaron este principio y lo relacionaron con el uso del aire, del agua y de la tierra para el bienestar y la preservación del mundo.

$\mathrm{El}$ aire era concebido como poseedor de influencia directa sobre la salud. En las notas a su edición hebrea del Régimen de Salud, S. Müntner apuntó veintitrés referencias de la literatura talmúdica y postalmúdica sobre este tema (38). Como se ha visto, en el Talmud aparece la noción de mazikin, espíritus causantes de enfermedad que viajan por el aire. También aparece en el Talmud la idea que sostiene que los sitios elevados son más saludables que los llanos y deprimidos en razón de la calidad de su aire. Aparece aquí una notable coincidencia entre las fuentes talmúdicas y los escritos de Galeno.

MM suscribe la opinión de Hipócrates en relación a los vientos. Para la escuela hipocrática los vientos se clasificaban en tres grupos: 
$1 .^{\circ}$ Los mejores: provenientes del litoral.

2. Los intermedios: provenientes de las montañas.

3. Los peores: provenientes de ríos, campos o lagos.

Empero, no cabe duda que debe haber tenido en cuenta también ciertos pasajes del Talmud donde el viento del norte es considerado una suerte de purificador del cielo (39) y necesario para la existencia del mundo (40). MM insistirá sobre los vientos y su relación con la salud al indicar hacia donde deben estar orientadas las viviendas para ser consideradas saludables, como ya se ha visto (41).

En un brillante trabajo Newmyer ha detallado la íntima relación entre la climatología de los escritos médicos griegos clásicos y la climatología médica del Talmud (42) En ambas escuelas se exigía al médico conocer las condiciones climáticas del lugar donde iba a trabajar antes de hacerlo. El texto Aires, aguas, lugares es un manual que brinda al médico consejo sobre cómo afrontar situaciones desconocidas. Los judíos del período talmúdico conocieron y valoraron estos escritos ya que les fueron útiles para hacer frente a situaciones desconocidas en la diáspora. Los maestros del Talmud aconsejaban a los médicos adelantarse a conocer el clima local si eran llamados a atender enfermos en ciudades extrañas (43). No es raro, entonces, que MM haya incluido en su Mishné Torá la indicación de hacerse atender siempre por médicos residentes en el lugar.

El médico cordobés se ocupó también de la eliminación y descarte de residuos biológicos. Basado en otro versículo bíblico (44) codificó la prohibición de defecar en el campamento (45). En las concentraciones militares debían designarse lugares especiales, fuera de los límites habitados, para defecar. Además, cada soldado debía llevar una estaca en su mochila; al sentir deseos defecatorios debía cavar un pozo y luego cubrir con tierra las heces, evitando que éstas quedasen expuestas a la intemperie. Los cadáveres de las reses y las tubas debían estar fuera de los límites del área poblada. Las curtiembres debían instalarse sólo en el lado este de la ciudad para evitar que el viente dispersase los olores nauseabundos que emanan de ellas. Las trilladoras debían ser alejadas de la ciudad para evitar que el viento esparciera la paja y dañara a los habitantes. Y si la trilladora fuera instalada dentro de un domicilio particular, debía verificarse que hubiera una distancia de terreno no menor a cincuenta cúbitos a la redonda para evitar la dispersión de la paja por el viento y la consiguiente molestia o daño a los vecinos. 


\section{Conclusión}

Del análisis de los textos precedentes surge que los conocimientos que MM tenía sobre climatología médica provenían de la medicina clásica grecorromana y de las fuentes bíblicas y talmúdicas. No existen evidencias suficientes para sostener que MM haya innovado en este campo, es decir, producido conocimientos nuevos y originales sobre este tema; antes bien, aparece como un buen organizador-clasificador-sistematizador del conocimiento médico preexistente.

En los escritos de MM se conjugan los enfoques diferentes de la medicina grecorromana clásica y de la medicina hebrea clásica. La primera luchó por separar la ciencia de la magia y de la religión; de esta forma, los procesos de salud y enfermedad podían ser estudiados racionalmente sin asumir ninguna clase de intervención divina. El hombre, en tanto ser racional, puede dominar el ambiente. En los escritos hebreos las cuestiones médicas en general -y las cuestiones ambientales tratadas aquí en particular - son vistas como inseparables de consideraciones religiosas y morales; entre ellas, la idea de una misión del hombre sobre la tierra y su condición de agente responsable del mantenimiento de la vida en el mundo que comparte con otras criaturas. Ambos enfoques revelan un agudo sentido de respeto por la naturaleza, al cual llegan por motivaciones diferentes. MM compartió ambas visiones ý, sin innovar en ninguna de ellas, supo organizar las opiniones de ambas escuelas y armar un cuerpo único de climatología médica completo, útil y racional.

\section{NOTAS}

(1) Múntner, S., ed. (1963). Rabenu Moshé ben Maimón. Sefer Hanhagat Habriut. Segunda edición. Jerusalén, pp. 65-68.

(2) Múntner, S., ed. (1940). Rabenu Moshé ben Maimón. Ktavim Refuiím. Sefer Hakatzeret o Sefer Hamis'adim. Primera edición. Jerusalén, pp. 33-34.

(3) El texto que aparece el Régimen de Salud (c1198) habría sido tomado por MM de su Tratado del Asma (ca. 1190). Las fechas de redacción de algunos obras de MM son inciertas. Se toman como válidas las fechas de redacción de ambos trabajos según la opinión de Romano y Rosner. Véase: Romano, D., ed. (1986). Maimónides y su época. Córdoba, p.p. 117-118; y Rosner, F. (1965). «The hygienic principles of Moses Maimonides». Journal of American Medical Association 194, 1352-1354, p. 1352.

(4) Müntner (1940), p. 16. 
(5) Pneuma y spiritus, en singular. Denominaciones griega y latina del mismo concepto.

(6) Rosner, F., trad., ed.; Múntner, S., trad., ed. (1973). Studies in Judaica. The Medical Aphorisms of Moses Maimonides.Vol. I y II. Segunda edición. Nueva York, p. 128.

(7) Laín Entralgo, P. (1979). Historia de la medicina. Barcelona, p. 86.

(8) Rosner, F. (1968). «Moses Maimonides' Responsum on Longevity». Geriatrics. Vol 23. 170-178; pp. 173-175.

(9) ROSNER, MÚNTNER (1973), p. 60.

(10) ROSNER, MÜNTNER (1973), p. 163.

(11) Rosner, G. (1958). A history of public health. Nueva York, p. 34. Citado por: Newmyer, S. (1984) «Climate and health: Classical and Talmudic perspectives». Judaism $33,426-438$, p. 430.

(12) JoNES, W., trad. (1923). Hippocrates. Airs waters places: Vol. I. Primera edición. Londres, p. 75.

(13) JoNES, W., trad. (1923), p. 77.

(14) Hacia el este.

(15) JONES, W., trad. (1923), p. 81.

(16) Hacia el oeste.

(17) JoNES, W., trad. (1923), p. 83.

(18) Newmyer, S. (1984), p. 432.

(19) GreEn, R. trad. (1951). Galen. Galen's Hygiene. De Sanitate Tuenda. Sprinnfield, p. 35-36. Citado por: Newmyer, S. (1984), p. 434

(20) Recuérdese que, de acuerdo con la tradición hebrea, la tierra de Israel no fue cubierta por las aguas del Diluvio, y sí lo fue el resto del mundo conocido. También debe tenerse presente que en la legislación bíblica y postbíblica el contacto de una persona con un cadáver causa la impureza de la misma.

(21) Mazikin. Esta palabra - con la declinación del plural -in, típica del hebreo mishnaico- está emparentada con nezek (daño), lehazik (dañar). Significaría aproximadamente «los que provocian daño».

(22) El pensamiento judío ortodoxo considera que el Pentateuco o Torá es la ley dada por divinidad al pueblo judío; que es la expresión de la voluntad de Dios y verdad absoluta. Como se trata de un texto escrito, es llamada Ley Escrita. Esta Ley Escrita contiene numerosos pasajes oscuros y confusos que dieron lugar al surgimiento de comentarios y explicaciones, transmitidos a través de las generaciones, y que por el hecho de ser de transmisión oral llevaron el nombre de Ley Oral. Alrededor del año 200 d.C. se decidió colocar por escrito por primera vez una síntesis de la Ley Oral, tarea encabezada por Rabí Judá el Príncipe y llevada a cabo en la tierra de Israel. Esa síntesis, distribuida en seis tomos, lleva el nombre de Mishná.

(23) Sotá 48b.

(24) Pirké Avot 5:9.

(25) Ketubot 104a.

(26) Shabat 63a.

(27) Mishná Babá Batrá 26b.

(28) Véase nota (22).

(29) Mishná Babá Batrá 24b. 
(30) Como la Mishná no era suficientemente clara y requería a su vez de interpretación, los rabinos continuaron discutiéndola y estudiándola. Aproximadamente en el año 400 d.C. los rabinos de Israel redactaron por escrito sus comentarios, los cuales recibieron el nombre de Guemará; la Mishná única y la Guemará redactada en Israel llevan, en conjunto, el nombre de Talmud de Jerusalén. Alrededor del año 500 d.C. los rabinos radicados en Babilonia procedieron también a poner por escrito sus comentarios, los cuales también recibieron el nombre de Guemará; la Mishná única y la Guemará de Babilonia llevan, en conjunto, el nombre de Talmud de Babilonia. El Talmud más utilizado es el babilónico, probablemente por ser más completo que el de Jerusalén. Tal es así que, cuando se menciona el Talmud a secas, se hace referencia al Talmud de Babilonia; para referirse al otro debe aclararse "Talmud de Jerusalén»o mediante su abreviatura convencional «T. J.»

(31) Babá Batrá 34b.

(32) Laín Entralgo, P. (1979), p. 112.

(33) Laín Entralgo, P. (1979), p. 89.

(34) Laín Entralgo, p. (1979), p. 86

(35) García Ballester, L. (1982), Galeno. Madrid. Citado por: Lain Entralgo, P., (1979), p. 89.

(36) MÜNTNER, S. Citado por: RosneR, F., en su manuscrito dactilografiado en inglés del Régimen de Salud de Maimónides, p. 99 y siguientes.

(37) Deuteronomio 20:19.

(38) MÜNTNER, S., trad. (1963), p. 67 y 68. Véanse allí notas 22-28b.

(39) Brajot 59a.

(40) Babá Batrá 25a.

(41) Véanse notas (1) y (2).

(42) Newmyer, S. (1984), p. 432.

(43) Babá Kamá 85a.

(44) Deuteronomio 23: 13-15.

(45) En el contexto de este pasaje bíblico la palabra "campamento» sería equivalente a "área habitada».

\section{BIBLIOGRAFÍA}

BoRTz, J., (1991); Maimónides. Biografía crítica y alqunos aspectos metodológicos, Buenos Aires, 53 p. Inédito. Trabajo que obtuvo el Premio Anual de Historia de la Medicina 1991.

Bortz, J., (1993); Medicina preventiva y psicosomática en los escritos de Maimónides, Tesis de Doctorado en Medicina, Buenos Aires, 721 p. Inédito. (Padrino de tesis: Prof. Dr. Alfredo G. Kohn Loncarica).

CASTIGlioni, A., (1941). Historia de la medicina, primera edición, Barcelona, 906 p.

GARRISON, F., (1960). An introduction to the history of medicine, cuarta edición, segunda reimpresión, Philadelphia, $966 \mathrm{p}$.

Laín Entralgo, P., (1979). Historia de la medicina, primera edición (reimpresión), Barcelona, $722 \mathrm{p}$.

Asclepio-Vol. XLVI-2-1994 
MARGALIT, D., (1962). Jajmei Israel ke-rofim, Jerusalén, 222 p.

ORIAN, M., (1984). Maimónides. Vida, pensamiento y obra, Barcelona, 430 p.

PeláeZ del Rosal, J., ed., (1991). Sobre la vida y la obra de Maimónides. Actas del I Congreso Internacional (Córdoba, 1985). Córdoba, 605 p.

Romano, D., ed., (1986). Maimónides y su época, Córdoba, 183 p.

Rosner, F., (1977). Medicine in the Bible and the Talmud. New York, 247 p.

Rosner, F., (1984). Medicine in the Mishneh Torah of Maimonides, primera edición, New York, $325 \mathrm{p}$.

Sigerist, H., (1967). A history of medicine. Vol. I. Primitive and archaic medicine. New York, $564 \mathrm{p}$.

TARgarona Borrás, J., trad., (1987). Moseh ben Maimón. Maimónides. Sobre el Mesías. Carta a los judios del Yemen. Sobre astrología. Carta a los judios de Montpellier. Biblioteca Nueva Sefarad. Vol. I. Barcelona, 268 p.

WeIL, G., trad., (1953). Maimonides: Uber die Lebensdauer. Basel-New York, 59 p. 